\title{
Prueba múltiple para inmunidad celular en niños con infecciones recurrentes
}

\author{
Arnoldo Quezada L. ', 2 ; Hilda Anderson I. ${ }^{3}$; \\ Viola Pinto $S .{ }^{1,2}$; Jorge Rodríguez T. ${ }^{4}$
Multitest for cell-mediated immunity in children with recurrent infections

\begin{abstract}
Cell-mediated immune response was evaluated by multitest for cutaneous delayed hypersensitivity with seven recall antigens and a glycerol diluent control in children with recurrent infections. Fifty patients, aged 4.9 years mean (range 1 to 14 vears), were selected by a score based on frequency, localization, severity and etiology of infectious episodes (Hosking) 13 . Results were confronted with those obtained from 21 healthy children aged 4.4 years mean \{range 1 to 4 years, $p$ : ns\}. Patients had lower number of positive reactions $\{2.06$ vs 3.95$\}$ and lower sum up diameter of all positive indurations $(7.29$ vs 13.69$)$ than controls ( $p<0.01)$. Responses to tetanic, diph teric, tuberculin and Protaus mirabilis antigens were significantly lower in patients versus controls imagnitudesi. Anergy was detected in $20 \%$ of patients. Diminished cellular immune response in children with recurrent infections may be adquired and transient. This alteration may worsen their pathological conditions or it mat be the conse. quence of multiple infectious episoder.
\end{abstract}

(Key words: Immunity. cellular; hypersensitivity. delayed: skin tests, immunologic deficiency svndromes, intections.)

Las infecciones recurrentes con frecuencia mayor que lo esperado se han descrito como características habitualmente presentes y altamente sospechosas de deficiencia funcional del sistema inmune ${ }^{1,2}$. Este puede presentar altera. ciones en cualquiera de sus componentes celulares y humorales ${ }^{3-5}$. La inmunidad celular juega un rol central en la regulación $y$ eficiencia de la respuesta inmunitarias. Entre los métodos de evaluación más útiles de la inmunidad celular se cuentan las pruebas de hipersensibilidad retardada $^{2,7,8}$. Para superar condiciones de falta de

1. Departamento de Pediatría Sur. Facultad de Medicina, Universidad de Chile.

2. Unidad de Inmunología. Hospital Dr. Exequiel González Cortés.

3. Enfermera Universitaria. Departamento de Pediatria Sur. Facultad de Medicina, Universidad de Chile.

4. Master en Estadistica y Matemática. Departamento de Salud Pública, Campus Sur. Facultad de Medicina, Universidad de Chile.

Proyecto M-2703 D.T.I. Univergidad de Chile. exposición previa en el niño, se recomienda el uso de varios antigenos. La prueba múltiple de hipersensibilidad retarda da ha sido estandarizada en estudios clínicos y experimentales y se han establecido valores de referencia normal en población sana adulta $\mathrm{e}$ infantil en publicaciones extranjeras ${ }^{9-12}$.

El objetivo del presente trabajo ha sido eva. luar la respuesta inmune celular en niños con infecciones recurrentes mediante la aplicación de una prueba cutánea múltiple de hipersensibilidad retardada y compararla con niños sanos.

\section{Material y Método}

El grupo de estudio, pacientes, estuvo constituido por niños que consultaron por infecciones recurrentes en la unidad de inmunología del Hospital Dr. Exequiel González Cortés entre enero de 1990 y novjembre de 1991 , a los cuales se aplicó un sistema de calificaciôn de acuerdo a la localización, severidad, frecuencin y etiología de los episodios infeccjosos, seleccionándose así 50 niños (28 varones) con edad promedio de 4,9 años (márgenes 1 a 14 años), que obtuvieron puntuaciones mayores de 20 según el esquema propuesto por Hosking ${ }^{13}$. Se excluyeron aquellos niños con inmuno- 
deficiencias secundarias. El grupo control estuvo constituido por 21 niños (11 varones), el promedio de cuyas edades era 4,4 antos (márgenes 1 a 14 años), sanos comparables, controlados en un consultorio de atención primaria del Servicio de Salud Metropolitano Sur. Todos los nirios (pacientes y controles) tenian sus irmunizaciones completas de acuerdo a su edad. A los padres y a los niños, cuando correspondia, se les informó sobre el objetivo del estudio y sobre el procedimiento a emplear y se obtuvo su consentimiento previo a la incorporación. En todos los nif̃os se practicó prueba múltiple (Multitest CMI, Instituto Merieux), mediante un dispositivo de multipuntura precargado con 7 ant ígenos (tetánico, diftérico, antígenos de Streptococcus grupo C, tuberculina, ant ígeno de Candida albicans, Trichophyton mentagrophytes y Proteus mirabilis) y un control de glicerina $70 \%$, que se aplicaron en forma simultánea en la cara anterior de un antebrazo y se leyó la respuesta cutánea de induración a las 48 horas, de acuerdo a las indicaciones del fabricante $y$ a las comunicaciones previas ${ }^{9-12}$. Se determinó el número de reacciones positivas para cada antígeno (induración mayor de $2 \mathrm{~mm}$ ) $\mathrm{y}$ el diámetro de cada una de las reacciones positivas obteniéndose una suma de diámetros de respuestas positivas para cada individuo. Se consultó sobre reacciones deșagradables.

En el análisis estad ístico se utilizó la prueba $t$ de Student para muestras independientes y prueba $\mathrm{Z}$ de diferencia de proporciones.

\section{Resultados}

No hubo diferencias estadisticamente significativas para la edad y el sexo entre ambos grupos $(p>0,05)$. Por orden de frecuencia, las infecciones que presentaban los pacientes fueron de ubicación cutánea (piodermitis, abscesos, celulitis), respiratorias bajas (neumonias, pleuroneumonías), septicemias, digestivas, ganglionares, osteoarticulares y otras (principalmente meningitis). En 24\% de los episodios infecciosos se confirmó un agente etiológico, siendo los más frecuentes: estafilococos, estreptococos, bacterias gram negativas y yirus.
En la tabla 1 se muestran jos resultados de la prueba cutánea múltiple. Se observan diferencias estadisticamente significativas entre pacientes y sanos respecto al número promedio de antígenos en que se obtuvo respuesta positiva $(2,06$ vs 3,$95 ; \mathrm{p}<0,01)$ y en cuanto a los promedios de la suma de diámetros de las respuestas positivas entre ambos grupos (7,29 vs 13,69 ; $\mathrm{p}<0,01)$. Entre los pacientes hubo 10 niños (20\%) con respuesta nula (anérgicos), observación que no se presentó en ningún sano.

En la tabla 2 se describen, por separado, las respuestas positivas para cada antígeno de la prueba cutánea múltiple. Se aprecian diferencias estadísticamente significativas entre pacientes y sanos en la respuesta a antígenos de tétanos ( $p<0,01)$, tuberculina $(p<0,001)$ y Proteus ( $p<0,001)$ al considerar los promedios y por. centajes. Para el antígeno difteria no existen diferencias estadísticamente significativas entre los grupos al comparar los promedios de la reacción positiva $(p>0,05)$; sin embargo, los porcentajes de positividad son significativamente mayores en los niños controles $(p<0,05)$. Destaca, además, la escasez de respuestas positivas obtenidas en ambos grupos para antígenos de Streptococcus y Trichophyton. En un niño normal de 1 año 9 meses de edad se observó una reacción flictenular de $5 \mathrm{~mm}$ en respuesta al antígeno tuberculina. Esta única reacción desagradable fue leve, autolimitada y no requirió medidas adicionales.

\section{Comentario}

La reactividad cutánea de hipersensibilidad retardada de riños que presentan infecciones a repetición resultó significativamente disminuida al compararlos con niños sanos. Esta menor reac-

Tabla 1

Respuesta cutánea a prueba múltiple

\begin{tabular}{|c|c|c|c|}
\hline & $\begin{array}{c}\text { Grupo } 1 \\
\text { n: } 50 \\
\bar{x} \pm \mathrm{DE}\end{array}$ & $\begin{array}{c}\text { Grupo } 2 \\
n: 21 \\
\vec{x} \pm \mathrm{DE}\end{array}$ & $\mathrm{p}$ \\
\hline n de ant ígenos positivos & $2,06 \pm 1,38$ & $3,95 \pm 1,02$ & 0,01 \\
\hline Suma de diámetros & $7,29 \pm 5,88$ & $13,69 \pm 4,33$ & 0,01 \\
\hline Anérgicos & $10 / 50$ & $0 / 21$ & \\
\hline
\end{tabular}


Tabla 2

Respuesta cutánea positiva por ant ígeno

\begin{tabular}{|c|c|c|c|c|c|c|c|}
\hline \multirow[b]{2}{*}{ Antígeno } & \multicolumn{3}{|c|}{ Grupo 1} & \multicolumn{3}{|c|}{ Grupo 2} & \multirow[t]{2}{*}{$\mathrm{p}$} \\
\hline & $\mathrm{n}$ & $\%$ & $\overrightarrow{\mathrm{x}} \pm \mathrm{DE}$ & n & $\%$ & $\overline{\mathbf{x}} \pm \mathbf{D E}$ & \\
\hline Tétanos & 27 & 54,0 & $2,15 \pm 2,36$ & 19 & 90,5 & $3,57 \pm 1,74$ & 0,01 \\
\hline Difteria & 14 & 28,0 & $0,94 \pm 1,78$ & 12 & 57.1 & $1,81 \pm 1,90$ & 0,05 \\
\hline Streptocaccus & 3 & 6,0 & $0,16 \pm 0,76$ & 4 & 19,0 & $0,43 \pm 0,93$ & ns \\
\hline Tuberculina & 18 & 36,0 & $1,61 \pm 2,41$ & 18 & 85,7 & $3,31 \pm 1,79$ & 0,001 \\
\hline Cándida & 26 & 52,0 & $1,64 \pm 1,77$ & 14 & 66,7 & $2,28 \pm 1,97$ & ns \\
\hline Trichophyton & 0 & 0,0 & $0,00 \pm 0,00$ & 1 & 4,8 & $0,10 \pm 0,44$ & ns \\
\hline Proteus & 15 & 30,0 & $0,81 \pm 1,32$ & 15 & 71,4 & $2,00 \pm 1,41$ & 0,001 \\
\hline
\end{tabular}

tividad se expresó en menor número y magnitud de las respuestas desencadenadas por los antígenos probados. En este mismo sentido debemos destacar entre los pacientes la elevada proporción de niños que no evidenciaron respuesta y que, por lo tanto, deben ser considerados anérgicos. En la interpretación de estos resultados deben tenerse en cuenta la exposición a los antígenos usados en la prueba, ya sea en forma natural o a través de vacunación, la inmunogenicidad de los preparados inoculados, la respuesta elaborada por las células inmunocompetentes, la expresión cutánea de esta respuesta y la participación de factores bloqueadores. Las condiciones de exposición y la inmunogenicidad deben ser com. parables en ambos grupos si se consideran la homogeneidad biodemográfica de los grupos estudiados y la estandarización del procedimiento. Las diferencias encontradas deben atribuirse por lo tanto a condiciones diferentes en la respuesta inmunitaria que en este caso compromete a linfocitos y macrófagos. En el grupo de estudio se descartó la presencia de inmunodeficiencias congénitas y las causas conocidas de inmunodefíciencia secundaria, por lo tanto debemos suponer que esta condición de defíciencia de la respuesta inmune celular es adquirida y asociada a causas diferentes de las consideradas.

Entre los agentes capaces de alterar la inducción y la expresión de la respuesta inmune celular se ha documentado la acción de los virus $^{14,}$ 15. La llamada anergia postinfecciosa descrita clásicamente en enfermedades como el sarampión, la varicela y otras enfermedades graves, parece también operar en otras infecciones por virus más corrientes y habituales. Tam- bién es necesario considerar la falta de reactjvidad transitoria que pueden desencadenar las vacunas con virus vivos atenuados. En este estu. dio tuvimos especial cuidado en realizar las pruebas alejadas de la vacunación de este tipo.

No podemos asegurar si la falta o la reducción de la respuesta celular está condicionada por una falla en las células $T$, por alteraciones de los macrófagos que participan en la expresión de la respuesta, por la existencia de factores capaces de bloquear las interacciones necesarias entre ambos grupos celulares o por una combinación de esos mecanismos. $\mathrm{Si}$ analizamos con mayor detalle las respuestas obtenidas entre ambos grupos, observamos que las diferencias fueIon significativas para 4 de los 7 antígenos utilizados en la prueba múltiple. De éstos, tres corresponden a respuestas a antigenos vaccinales (tétanos, difteria y tuberculina) y el cuarto antígeno que marca diferencia en la reactividad entre pacientes y sanos corresponde a Proteus. No encontramos diferencia en las respuestas a antígenos de cándida, Streptococcus y Trichophyton. Con estos dos últimos antígenos el número de respuestas positivas fue muy bajo en los dos grupos de niños estudiados. Es probable que la antigenicidad del preparado en el primer caso y las condiciones de exposición en el segundo sean responsables de los bajos índices de respuesta observados para antígeno de Streptococcus y Trichophyton, respectivamente. La adecuada reactividad para antígeno de cándida, detectada en el grupo de pacientes, indica que no se trata de niños con inmunodeficiencia celular absoluta $y$, por lo tanto, se puede postular que el agente o factor desencadenante de la 
alteración de la respuesta inmune tiene cierta selectividad sobre algunos clones de linfocitos de memoria, o bien que existen factores bloqueadores, también con cierto grado de selectividad, que interfieren con la adecuada $y$ necesaria serie de eventos celulares y bioquími$\cos$ involucrados en la respuesta inmune celular.

Con los resultados obtenidos no podemos descartar que las alteraciones encontradas en la respuesta celular sean consecuencia de las múltiples infecciones sufridas por estos pacientes. Debemos señalar que no está definitivamente establecido cuál es el número de episodios infecciosos que pueden considerarse normales a las diferentes edades o según el sistema comprometido $y$, en base a esta normalidad, cuándo debería plantearse la necesidad de evaluación inmunologica por sospecha de deficiencia en los mecanismos de defensa, como causa de susceptibilidad aumentada a las infecciones. El motivo más frecuente para referir un niño a una clínica de inmunodeficiencia son las infecciones respiratorias, de las cuales las altas pueden ocurrir entre seis y diez veces durante el primer año de vida de un niño normal ${ }^{16,17}$. En países de sub. desarrollo relativo $y$ en poblaciones rurales se acepta que un individuo sano puede sufrir hasta siete episodios anuales de diarrea aguda dentro de la frecuencia habitual ${ }^{18,19}$ y en América Latina un promedio de cuatro por ano ${ }^{20}$. De acuerdo a estudios clínicos se requieren dos o más episodios de neumonía radiológicamente demostrada para considerarlas recurrentes ${ }^{21}$. En todo caso, cuando las infecciones se presentan en un solo aparato o localización deben descartarse las alteraciones anatómicas o funcionales locales como primera posibilidad cau$\mathrm{sal}^{22,23}$.

En este estudio hemos utilizado el esquema propuesto por Hosking ${ }^{13}$, que asigna puntos a los episodios infecciosos, según su severidad, localización, frecuencia y etiología para decidir la oportunidad del estudio inmunológico, previo descarte de alteraciones anatómicas locales e inmunodeficiencias secundarias. Creemos que este método ha sido útil para separar niños sanos de pacientes con alteraciones inmunitarias $y$, por lo tanto, recomendamos su uso para facilitar la decisión de someter a pruebas de laboratorio a un niffo con infecciones recurrentes.

La reactividad cutánea retardada como indicador del estado de actividad de la inmunidad celular ha sido utilizada como evaiuación pre- quirúrgica de pacientes oncológicos, en trastornos nutricionales y en pacientes con infecciones crónicas ${ }^{24-29}$. Las pruebas de hipersensibilidad retardada han empleado antígenos como tuberculina, toxoide tetánico, toxoide diftérico $y$ cándida con resultados positivos en un alto nú. mero de niños sanos y han demostrado buena correlación con otras pruebas de inmunidad ce. lular, tales como la estimulación de linfocitos in vitro (transformación linfoblástica) ${ }^{30-33}$. La reacción de multipuntura para tuberculina ha sido utilizada por más de 25 años ${ }^{3 a}$. En nuestros niños controles sanos, las respuestas positivas alcanzaron altos porcentajes para 5 de los $7 \mathrm{an}$ tígenos incluidos en la prueba cutánea múltiple (tétanos, tuberculina, Proteus, Candida y difteria), sin sujetos anérgicos entre ellos, a diferencia de lo descrito en otros estudios. Además, en el grupo control el promedio de la suma de los diámetros de las respuestas positivas fue mayor que en otros reportes ${ }^{11,12}$

La prueba cutánea múltiple es un método adecuado y de alto rendimiento para evaluar la respuesta inmune mediada por células y permite establecer diferencias en la capacidad de respuesta entre niños sanos y otros con infecciones recurrentes, más frecuentes que lo esperado.

\section{Resumen}

Con el objeto de evaluar la respuesta inmune celular en niños con infecciones recurrentes, seleccionados de acuerdo a una calificación asig. nada por frecuencia, localización, severidad y etiología de los episodios infecciosos, se aplicó una prueba múltiple con siete antígenos (diftérico, tetánico, tuberculina, de Candida albicans, de Streptococeus grupo C, de Proteus mirabilis $\mathrm{y}$ de Trichophyton mentagrophytes) para hipersensibilidad retardada en 50 pacientes cuya calificación fuese de 20 o más puntos, siguiendo los criterios de Hosking. Se excluyeron enfermos con inmunodeficiencias primarias y secundarias conocidas. Se compararon los resulta. dos con 21 niños sanos (controles). Los pacientes tuvieron respuestas significativamente menores $(\mathrm{p}<0,01)$ en cuanto al numero de reacciones positivas y la suma de los diámetros de las reacciones. Al analizar cada uno de los siete antígenos se obtuvieron diferencias significativas entre pacientes y controles en la respuesta frente a tétanos $(p<0,01)$, difteria $(p<0,05)$, tuber- 
culina ( $\mathrm{p}<0,001$ ) y antigenos de Proteus (p $<0,001$ ). Entre los pacientes hubo 10 ninos anérgicos (ausencia de respuesta a todos los antígenos probados), observación no encontrada entre los ninos sanos. La respuesta celular deprimida que presentan estos pacientes con infecciones recurrentes parece ser una condición adquirida y transitoria, que contribuye a su estado patológico, o bien puede ser consecuencia de los múltiples episodios in fecciosos.

(Palabras claves: Inmunidad celular, hipersensibilidad retardada, pruebas cutaneas, inmunodeficiencias, infecciones recurrentes.)

\section{Referencias}

1. Ammann A, Fudenberg $H$ : Immunodefictency Diseases. En: Fudenberg $H$ Basic and Clinical Immunology, Los Altos, USA: Lange Medical Publications $1978 ; 391-421$.

2. Johnsion $R$ : Recurrent bacterial infections in children. N Engl J Med 1984; 310: $1237-1243$.

3. Rosen $F$, Cooper $M$, Wedgwood $R$ : The primary immunodeficiencies (first of two parts). N Engl J Med 1984; 311: 235-242.

4. Rosen $F_{+}$Cooper $M$, Wedgwood $R$ : The primary immunodeficiencies (second of two parts). N Engl J Med 1984; $311:$ 300-310.

5. Stichm $R$, Fulginiti $V_{i}$ Immunologic disorders in infants and children. Philadelphia Ed. WB Saunders Co. 1989.

6. Ogra P. Jacobs $D$ : Regulation of the immune response. Basel Ed. Karger 1983.

7. Stites $D$ : Clinical laboratory methods of detecting cellular immune function. En: Fudenberg $\mathbf{H}$, Basic and Clinical Immunology. Los Altos, USA: Lange Medical Publications. 1978: 375-388.

8. Spitler $L$ : Delayed hypersensitivity skin testing. In: Rose R, Friedman, Eds. Manual of Clinical Immunology. Washington, Amerjcan Society for Microbiology, 1976;53.

9. Kniker $W$, Anderson $C$, Roumionizeff $M$ : The multitest system: A standarized approach to evaluation of delayed hypersensitivity and cellmediated immunity- Ann Allergy 1979; 43: 7379.

10. Kniker W. Anderson C. McBryde J, Roumiontzeff $M$. Lesourd $B$ : Multitest CMI for standarized measurement of delayed cutaneous hypersensitivity and cell-inediated immunity. Normal values and proposed scoring system for healthy adults in USA. Ann Allergy 1984:52:75-82.

11. Corriel $R$, Kniker $W$, Mceiryde $J$, Lesourd $B$ : Cell-mediated immunity in school children asses. sed by multitest skin testing. AJDC 1985; 139: 141-146.

12. Kniker $W$, Lesourd $B$, McBryde $J$, Corriel $R$ : Cell-mediated inmunity assessed by multitest
CMI skin testing in infants and preschool children. AJDC 1985; 139: 840-845.

13. Hosking $C$, Robertson $D$ : The diagnostic approach to recurrent infections in childhood. Clin Immunol Allergy $1981 ; 1 ; 631-639$.

14. Ammonn $A$, Fudenberg $H$ : Enfermedades por inmunodeficiencia. En: Fudenberg $H$, Stites DP: Inmunología Clínica. México. Ed. El Manual Moderno $1982 ; 418-451$.

15. Southem $P$, Oldstone $M$ : Medical consequences of persistent viral infection. N Engl J Med 1986; 314: 359-36?

16. Siegel $S$ : Recurrent and chronic upper respiratory infections and chronic otitis media. En: Bierman $C W$, Pearlman DS: Allergic diseases from infancy to adulthood. Philadelphia Ed. WB Saunders Co. 1988: 717-729

17. Beard $L$, Maxwell $G$, Thong $Y$; Immunocompetence of children with frequent respiratory in. fections. Arch Dis Child 1981;56: 101-105.

18. Black $R$, Brown $K$, Becker $S$, Abdul Alim $A$. Hug $I:$ Longitudinal studies of infectious diseases and physical growth of children in rural Bangladesh. Incidence of diatrhea and association with know pathogens. Am J Epidemiol 1982; 115: 315-324.

19. Mato L, Urutia $J$, Simhon $A$ : Infectious agents in acute and chronic diarrhea of childhood. En: Lebenthal E: Chronic diarrhea in children. New York Ed. Raven Press 1984; 237.252.

20. Dufou $G$ : Clínica del síndrome diarreico agudo en el riño. En: Meneghello J: Diálogos en Pediatr ía III. Santigo Ed. Publicaciones Técnicas Medite. rráneo Ltda. 1990: 205-216.

21. Kjetlman $B$ : Bronchial asthma and recurrent pneu. moria in children. Acta Paediate Sand 1967; 56: $651-659$.

22. Quezade A: Infecciones recurrentes. En: Meneghelo J: Diálogos en Pediatría V. Santiago. Ed. Publicaciones Técnicas Mediterráneo Ltda. 1992; 31-39.

23. Quczada A: Enfoque clínico del rin̄o con infecciones a repetición. Rev Chil Pediatr 1981; 52: 520-523.

24. Galant S, Flod N, Shimizu I, Granger G, Groncy C: Relationship between cutaneous delayed hyper. sensitivity and cell-mediated immunity in vitro responses assessed by diphteria and tetanus toxoids. J AlJergy Clin Immunol 1977; 60: 247-253.

25. Tomar $R$, Taylor $F$, Greeng $R$ : Delayed hypersensitivity to SK-SD: In vitro lymphocyte study. J Immunol 1972; 108: $231-235$.

26. Meakins $J$. Pietsch $J$, Busenik $O$ : Delayed hypersensitivity: Indicator of adquired failure of host defenses in sepsis and trauma. Ann Surg 1977; 186: 241-250.

27. Johrson $W$, Urich $F$, Meguid M: Role of delayed hypersensitivity in predicting postoperative morbidity and mortality. Am J Surg $1979 ; 137: 536$ 542.

2B. Daly $J$, Dudrick $S$, Copeland $E$ : Intravenous hyperalimentation: Effect on delayed cutaneous hypersensitivity in cancer patients. Antn Surg 19.80; 192: $587-592$. 
29. Twomey $P$, Ziegier $D$, Rombeau $J$ : Utility of skin testing in nutritional assessment; $A$ critical review. JPEN $1982 ; 6$ : $50-58$.

30. Fronz $M$, Corelih $J$, Golant $S$ : Cutaneous dejayed hypersensitivity in a healthy pediatric population; Diagnostic value of diphteria-tetanus toxoids. J Pediatr 1976;88: 957-977.

31. Stecle $R$, Suttle D, Le Master P, Patterson $F$, Ca. noles $L$ : Screening for cell-mediated inmunity in children. AJDC 1976, 130 : 1218-1221.
32. Munoz A, Limbert D: Skin reactivity to Candida and streptokinase-streptodornase antigens in normal pediatric subjects: Influence of age and acute illness. J Pediatr 1977;91:565-568.

33. Borut T, Ank B, Gard S, Sttehm E: Tetanus toxoid skin test in children: Correlation with in yirro lymphocyte stimulation and monocyte chemotaxis. J Pediatr 1980;97: 567-573

34. Kravitz H, Burg $F$, Lawson $R$ : Skin testing with liquid old tuberculin with a multiple puncture technique. Pediatrics 1968;42: 465-470. 